\title{
Hybrid SVD Model for Document Representation
}

\author{
P. Kalpana, R. Kirubakaran, P. Tamije Selvy
}

\begin{abstract}
Document clusters are the way to segment a certain set of text into racial groups. Nowadays all records are in electronic form due to the problem of retrieving appropriate document from the big database. The objective is to convert text consisting of daily language into a structured database format. Different documents are thus summarized and presented in a uniform manner. Big quantity, high dimensionality and complicated semantics are the difficult issue of document clustering. The aim of this article is primarily to cluster multisense word embedding using three distinct algorithms (K-means, DBSCAN, CURE) using singular value decomposition. In this performance measures are measured using different metrics.
\end{abstract}

\section{Keywords : SVD, K-means, DBSCAN, CURE.}

\section{INTRODUCTION}

Every day, with tremendous development in the Internet, more than quintillion data were produced. The most significant advance in illustration experiences from unstructured text information, which is an objective of Natural Language Processing(NLP), is to change these unstructured content information into numerical vectors. This is known as the learning representation. In this article we concentrate on document representation teaching.

A document clustering scheme's objective is to minimize intra-cluster distance between documents while maximizing inter-cluster distance. A distance measure therefore lies at the core of document clustering. The wide range of papers makes it difficult to generate a particular algorithm that can function better for all types of datasets. Fuzzy Bag of Words(FBoW) is one of the efficient approaches to document clustering.

Fuzzy has three modules, which are recognized as a nonlinear knowledge based scheme, of fuzzy, de-fluzzy and inference. Fuzzy Word Bag is suggested to know more thick and powerful document representation encoding more semantics. FBoW replaces difficult mapping with fuzzy mapping[1], introduces vagueness in matching phrases with basic terms. In FBoW word embedding method is introduced to assess semantic similarity.Word significances can be encrypted in a vector and the semantic similarity between them can be evaluated by using cosine. TheFuzzy membership feature depends on the similarity between sentences and words.Fuzzy Bag of Word cluster is

Revised Manuscript Received on 14 August, 2019.

P. Kalpana, Assistant Professor, Department of Computer Science and Engineering1, Sri Krishna College of Technology, Coimbatore, Tamilnadu, India.(Email: kalpanapaulrajphd@gmail.com)

R. Kirubakaran, Assistant Professor, Department of Computer Science and Engineering, Kumaraguru College of Technology, Coimbatore, Tamilnadu, India.(Email: kirubakaran.r.cse@kct.ac.in)

Dr. P. TamijeSelvy, Professor, Department of Computing Science and Engineering, Sri Krishna College of Technology, Coimbatore, Tamilnadu, India.(Email: p.tamijeselvy@skct.edu.in). suggested based on FBoW. FBoWC utilizes word clusters as the basic concepts. Three variants named respectively fuzzy bag of word mean, fuzzy bag of word minimum, fuzzy bag of word maximum [1]. Fuzzy Bag of Words primary contribution:

1.FBoW model decreases sparsity, improves power and encodes more semantic data, adopts Fuzzy mapping in which word semantic similarity can be determined by value.

2.FBoWC generates lower-dimensional representation than FBoW.It is based on the similarity between phrases and cluster within the corpus. The resemblance can be regarded as the extent of one sentence which corresponds semantically to another word.

D1 : $\{$ "Dog bark on the sun" $\}$

D2 : $\{$ "Cat licks a kid." \}

If the FBoW uses the following basic terms:\{ "cat," "bark," "dog," "Kid"\}, the 2 sentences $\mathrm{D} 1=(\{1\},\{0\},\{7\},\{0\},\{8\},\{1\}) \& \mathrm{D} 2=(\{0\},\{7\},\{1\},\{1\},\{0\}$,

$\{8\})$ respectively.

The informative term sat is overlooked for phrase D1 owing to blurred mapping of BoW. In short, fuzzy model bag of words cannot capture semantic documentation,leading to bad results in the issue of classification and regression. In Fig 1. Shows the fuzzy mapping of words though it is better than BOW but still suffers from word semantic embedding's problems.

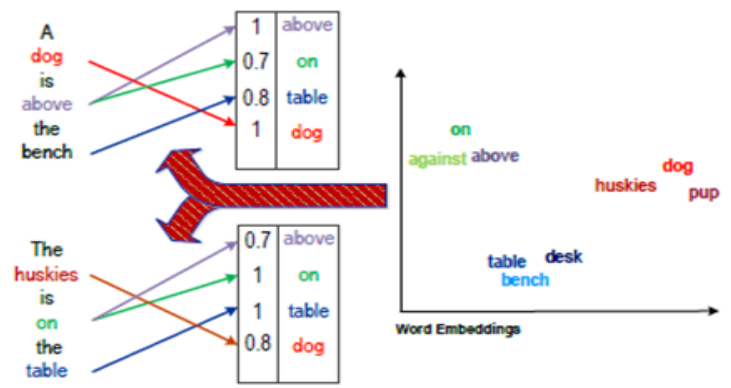

Figure 1. Fuzzy BOW

Clustering Learning Representation:

The fuzzy affiliation function is used to count the number of terms in a document.the predation of the FBag of Words document is incidation by $\mathrm{x}=[\mathrm{x} 1, \mathrm{x} 2, \mathrm{x} 3 \ldots . . \mathrm{xi}]$ where the $\mathrm{Zi}$ element is the sum of membership degree which all words correspond semantically to the term

$$
\mathrm{Zi}=\text { ci } \mathrm{E} \operatorname{ATi}(w j) x j s
$$

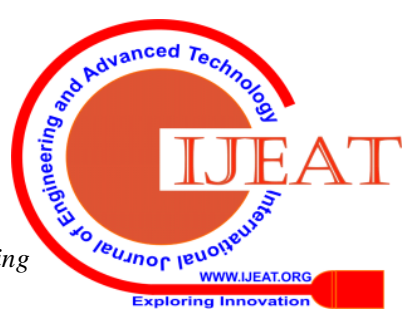


$\mathrm{w}-\mathrm{a}$ collection of all the phrases in the document.

$\mathrm{Ti}$ - I word

xjs-the occurrence amount of wj.

Clusters data grounded only on three variants (min, mean, $\max$ ), it clusters small quantities of data(min) or large quantities of data(max) so the mean varies significantly according to clusters.We suggest a hybrid SVD for document clustering in this paper. Fuzzy mapping by hybrid SVD(SVD-K means, SVD-DBSCAN,SVD CURE) to solve te restriction of the initial Fuzzy bag or words.Hybrid SVD introduces vagueness in maching phrases and baseline terms given in Figure 2.

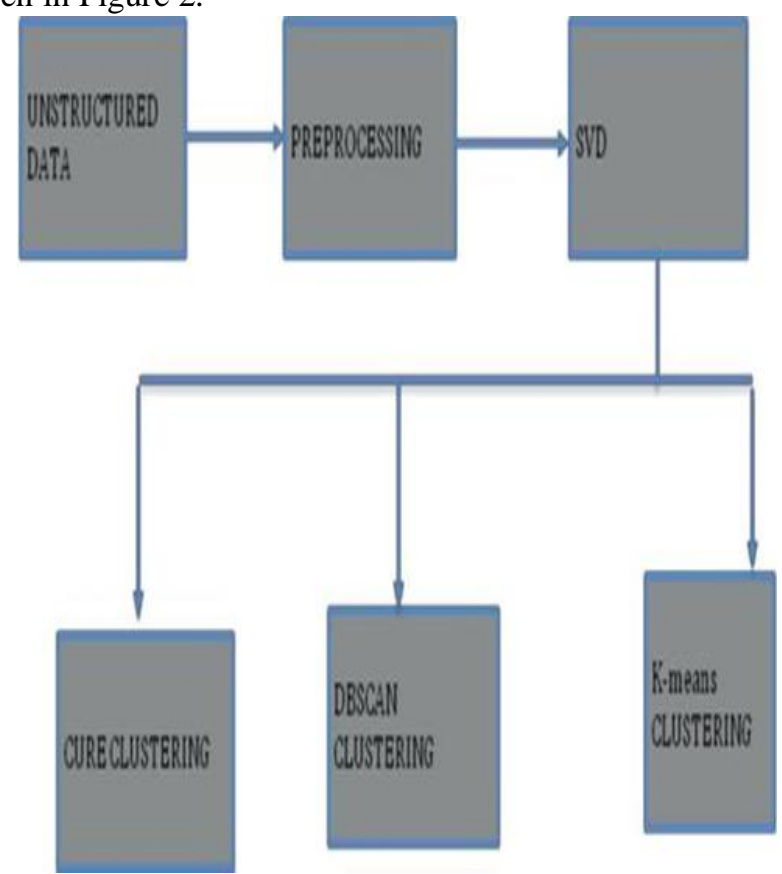

Figure 2. Hybrid SVD

\section{RELATED WORK}

\section{A. Document Pre-processing}

The purpose of pre-processing is to ignore all characters and conditions with bad document data that influence the quality of group descriptions[5]. The first method is to remove Stop Words that have no data and significance such as pronoun, preposition, etc... Stop words were automatically deleted by the TF-IDF vector that has the natural ability to remove stop words.

\section{B. (SVD)Singular Value Decomposition}

In uncontrolled linear minor problems, matrix ranking estimates and canonical correlation analysis, SVD is commonly used [2]. A big dataset is split into smaller ones each containing close-to-sense information. SVD constructs $\mathrm{n}$ dimensional abstract semantic space in which term and document are represented as vectors.

$\mathrm{A}=\mathrm{T}-1$ where the unit matrix is $\mathrm{T}=\mathrm{m}$.

$\mathrm{m}=\mathrm{n}$ diagonal matrix

$\mathrm{D}=\mathrm{n}$ nn unit matrix.

D* is the unitary matrix's conjugate transposition.

\section{Latent Semantic Analysis (LSA)}

LSA is a vector space model expansion that utilizes SVDs to split document into a larger size.Using truncated SVD,LSA can be used to decrease the document
dimension.Let A be MxN word matrix,LSA breaks up the matrix to $\mathrm{MxN}$ orthogonal matrix T,PxP orthogonal[first diagonal]matrix D-1 is the latent semantic word matrix.

\section{K-means}

It is an parsing algorithm in which the findings rely on the original value of centroids. The objective of using k-means is to group in k-clusters a collection of data with $\mathrm{k}$ values[4][8].K-means collects the vectors from the SVD and calculates the centroid values. It produces distinct outcomes for each stimulus, we run the technique several times until centroid values do not change[2]. It attempts to create intraclusters as comparable as possible. K-means large data clusters and produces high clusters.

\section{E. DBSCAN}

Density Based Spatial Clustering of Noise Applications is a process of grouping points together on a number of points close to each other based on a range measurement (Euclidean distance) and a minimum amount of points[12]. It also makes the points in low-density regions as outliers[3]. It is used to locate outliers and noise clusters of any form in the dataset. Two significant parameters are required for DBSCAN.

dbscan $=($ epsilon, minpts $)(2)$

\section{Epsilon (EPS)}

Epsilon characterizes the range of the area around a point $x$ called as neighborhood of $x$. It implies that if the separation between the two points is lesser or equivalent to the worth (eps)[8] then these focuses are said to be neighbors.

\section{Minimum Points (MINPTS)}

MinPts is the base number of dense locales. The base estimations of minPts must be 3 . On the off chance that the dataset is bigger, at that point the estimation of the minPts ought to be picked.By using these parameters, DBSCAN calculates the value of the cluster. DBSCAN collects the data set from SVD after the process and performs its process. The data set is grouped according to the parameter condition. In the first partition the eps value is found by defining the eps neighborhood and the clustering process takes place as the second partition with the use of minPts. Unlike other algorithms[7], In DBSCAN amount of clusters to be created is not needed to mention. DBSCAN can discover any cluster forms and recognize outliers.

\section{F.CURE}

CURE is a hierarchical algorithm for clustering that produces a equilibrium between centroid approaches and all point approaches. A fixed number of well-scattered points are selected in a cluster as representatives and those points take all the possible shape that the cluster might have[12].The cluster in each CURE stage are the closest pair of cluster. The outliers are most robust and clusters of large varieties and other non-sphere forms are identified. 


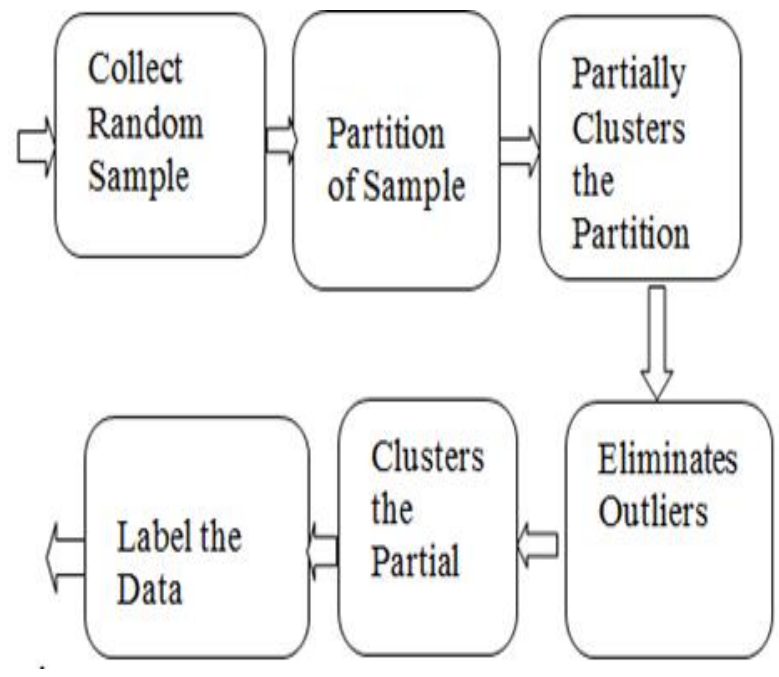

Figure 3. CURE Process

CURE is obtained by depicting every cluster by choosing well-dissected points from the clusters and reducing them by a given fraction to the middle of the cluster[6]. It makes it possible for CURE to adjust to non-sphere form geometry and helps to decrease the effect of outliers where more than one representative point per cluster exists[13].

In Figure 3. The CURE technique is discussed with a combination of random sampling and partitioning. A random sample will be taken from the dataset in the first partition and each partition will be partly clustered [11]. The cluster you want is yield from the partial cluster that is clustered in the second pass. The output proves that the performance generated by the clusters is much better than the algorithms that exist. A mixture of partitioning and random sampling makes it possible for not only to override other present algorithms, but also scale for large database without enabling the cluster quality. [10].

\section{RESULTS}

\section{A .Dataset Description}

In this paper we collected datasets from 20 newsgroups, which include a collection of 20,000 newsgroup documents, collection data sets, which are popular as a dataset for experiments in machine training techniques, such as text classification and text clustering.

\section{B. Data Characteristic}

100000 usenet articles were taken from 20Newsgroups.In this paper, we have used four characteristic from set.

\section{Experimental Results}

Measures of Hybrid SVD includes completeness, homogeneity, V-measure, Adjusted RandomIndex

\section{Homogeneity}

Homogeneity is the quality or condition that is uniform in the whole dataset.This is the primary method for checking the (identical)homogenous property of a document.

\section{Completeness}

Completeness is the state or condition of having all the necessary \& suitable document paths it checks whether or not all the paths are in the right position.

\section{V-Measure}

$\mathrm{V}$-measure is the mean for the grouping all components in a single cluster between homogeneity and completeness.

\section{Adjusted RandomIndex}

The randomindex is the used to number the pairs between 0\&1 in the same group.In figure 4 represents the hybridization of SVD with K-means,DBSCAN and CURE.

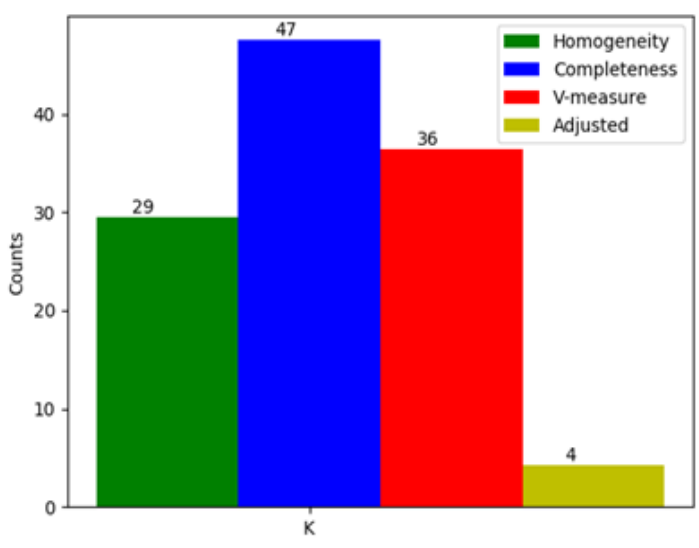

Figure 4. Hybrid SVD K-MEANS

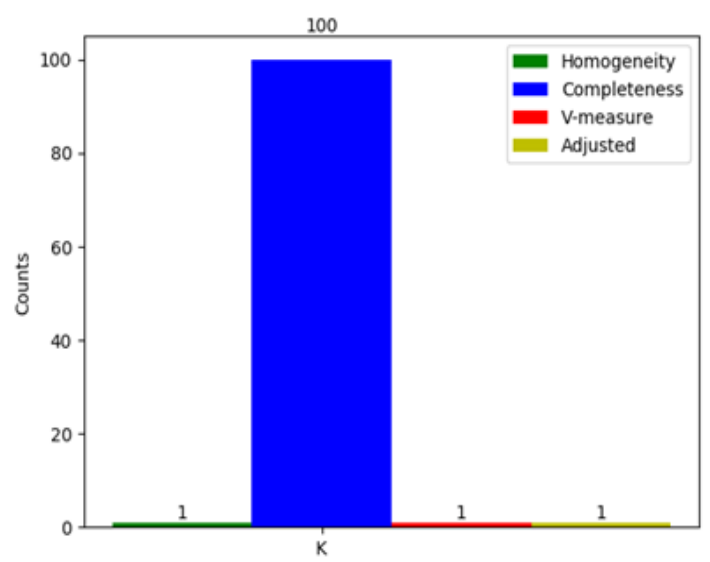

Figure 5. Hybrid SVD DBSCAN

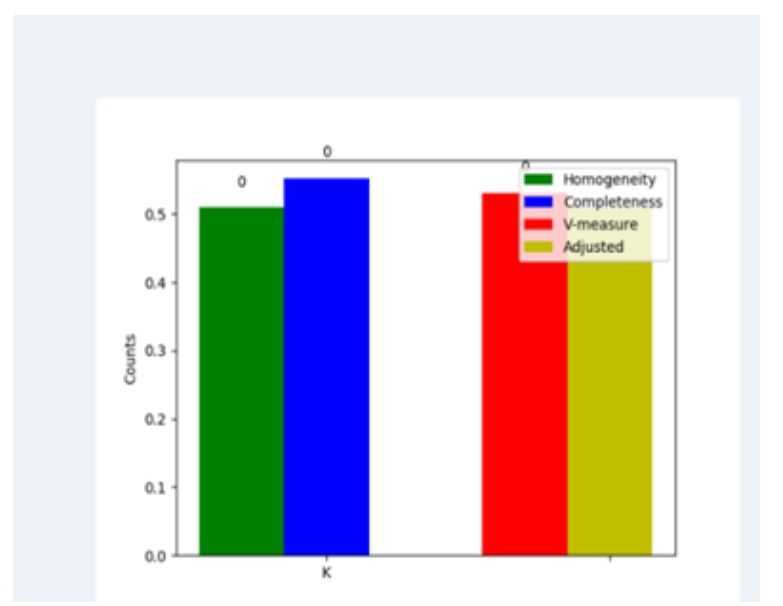

Figure 6. Hybrid SVD CURE 


\section{CONCLUSION}

The effects of document sampling result with different methods of vectorization are observed in this paper. The depiction of documents has a greater effect on classification and clustering outcomes because they capture more document semantics and decrease the issue of high dimensionality. CURE provides better results in clustering in above three algorithms, i.e. it provides greater precision. As a next step, we will be able to propose the effect of multi-sense word embedding.

\section{REFERENCES}

1. Rui Zhao and Kezhi Mao "Fuzzy Bag Of Words Model for document Representation" in IEEE transaction on Fuzzy System, vol.26,No. 2, April 2018.

2. Michael B. Cohen, Sam Elder, Cameron Musco, Christopher Musco, Madalina Persu" Dimensionality Reduction for k-Means Clustering and Low Rank Approximation" April 2015.

3. R.janai and Dr. S.Vijayarani "An Efficient Algorithm for document Clustering in Information Retrieval " Vol 4,Isuue XII, December 2016.

4. Michal Aharon, Michael Elad, and Alfred Bruckstein"KSVD: An Algorithm for Designing Overcomplete Dictionaries for Sparse Representation" IEEE Transaction On Signal Processing, Vol, 54, No. 11, November 2006

5. Paul S. Bradely and Usama M. Fayyad "Initial points for K-mean Clustering". In Proceedings of the 15th International Conference onMachine Learning(ICML98), 1998.

6. Kiri Wagsta, Claire Cardie, Seth Rogers, Stefan Scroed "Constrained K-means Clustering with Background Knowledge." Proceedings of the Eighteenth International Conference on Machine Learning, pages 577584, 2001.

7. A.Hotho, S.Staab and G.Stumme,"Wordnet improves text document clustering" In Proceedings of the SIGIR Semantic Web Workshop, Toronto, 2003.

8. Siamala Devi S, Shanmugam A., "An Integrated Harmony Search Method for Text Clustering using a Constraint based Approach", Indian Journal of Science and Technology, Vol 8(29), 73986, 2015.

9. Bjornar Larsen and Chinatsu Aone "Fast and Effective Text Mining Using Linear-time" In Proceedings of the fifth ACM SICKDD International Conference on knowledge Discovery and Data Mining, 1999.

10. Sethuramalingam, T. K., and B. Nagaraj. "A comparative approach on PID controller tuning using soft computing techniques." International Journal of Innovations in Scientific and Engineering Research (IJISER) 1, no. 12 (2014): 460-465.

11. D.D Lewis Reuters-21578 "Text Categorization text collection distribution" In proceedings of ACM SIGKIDD on 1999.

12. Lin "Divergence measures based on the Shannon entropy", IEEE transaction On information theory, 37(1):145-151-1991.

13. D. Arthur and S. Vassilvitsku" K-means - the advantage of careful seedings". In symposium on discerete algotithm, 2007.

14. D.Milne, O.Medelyan, and I.H.Witten. Mining domainspecfic thesauri from Wikipedia: A case study. In Proc. Of the International Conference on Web Intelligence (IEEE/WIC/ACM WI'2006),2006. 